speed, 1 to $50 \mathrm{mg}$ of PFA could be applied in each coating step.

13. The coated tubes were placed inside a quartz pipe $\mathbf{5 7}$ $\mathrm{mm}$ in diameter, fitted with end caps designed to hold the coated tubes in the center while they were being rotated. The quartz pipe was fitted into a temperaturecontrolled furnace and purged with scientific-grade $\mathrm{He}$ (total impurities $<1$ part per million) at a flow rate of $100 \mathrm{cc} / \mathrm{min}$ for $15 \mathrm{~min}$. The temperature was raised at a rate of $5.0^{\circ} \mathrm{C} / \mathrm{min}$ to the final temperature ( 473 to $873 \mathrm{~K}$ ) and held there for $120 \mathrm{~min}$. To ensure uniformity during pyrolysis, we rotated the tubes at $30 \mathrm{rpm}$.
14. The gas was introduced on the core (feed) side of the NPCM at a pressure of $300 \mathrm{kPa}$, and the shell side pressure response was measured continuously. The membrane module was evacuated and returned to atmospheric pressure on both the core and shell sides before the introduction of the next probe gas. All experiments were conducted at $295 \mathrm{~K}$.

15. J. W. Hutchinson and Z. Suo, Adv. Appl. Mech. 29, 63 (1991).

16. R. C. Reid, J. M. Prausnitz, B. E. Poling, The Properties of Gases and Liquids (McGraw-Hill, New York, ed. 4, 1987)
17. $P_{\mathrm{ss}}$ and $P_{\mathrm{cs}}(\mathrm{Pa})$ are the pressures on the shell side and core side of the tubular membrane, respectively; $t(\mathrm{~s})$ is time; $A\left(\mathrm{~m}^{2}\right)$ is the membrane area; $R\left(\mathrm{~m}^{3} \mathrm{~Pa} \mathrm{~mol}^{-1}\right.$ $\left.\mathrm{K}^{-1}\right)$ is the gas constant; $T(\mathrm{~K})$ is the temperature; $V_{\mathrm{ss}}$ $\left(\mathrm{m}^{3}\right)$ is the shell side volume; $\pi^{\prime}$ is the gas permeability $\left(\mathrm{mol} \mathrm{m}^{-1} \mathrm{sec}^{-1} \mathrm{~Pa}^{-1}\right)$; and $\delta(\mathrm{m})$ is the membrane thickness.

18. L. M. Robeson, J. Mem. Sci. 62, 165 (1991).

19. Supported by Department of Energy Office of Basic Energy Science, State of Delaware Research Partnership, and DuPont.

30 March 1999; accepted 9 August 1999

\section{Room Temperature Lasing at Blue Wavelengths in Gallium Nitride Microcavities}

\author{
Takao Someya, ${ }^{1 *}$ Ralph Werner, ${ }^{2}$ Alfred Forchel, ${ }^{2}$ \\ Massimo Catalano, ${ }^{3}$ Roberto Cingolani, ${ }^{4}$ Yasuhiko Arakawa ${ }^{1}$
}

\begin{abstract}
Lasing action has been demonstrated at blue wavelengths in vertical cavity surface-emitting lasers at room temperature. The microcavity was formed by sandwiching indium gallium nitride multiple quantum wells between nitridebased and oxide-based quarter-wave reflectors. Lasing action was observed at a wavelength of 399 nanometers under optical excitation and confirmed by a narrowing of the linewidth in the emission spectra from 0.8 nanometer below threshold to less than 0.1 nanometer (resolution limit) above threshold. The result suggests that practical blue vertical cavity surface-emitting lasers can be realized in gallium-nitride-based material systems.
\end{abstract}

Blue nitride-based semiconductor laser diodes (LDs) and light-emitting diodes (LEDs) have been developed over the past few years $(1-13)$ and are having a large impact on industry as well as on fundamental research. Blue vertical cavity surface-emitting lasers (VCSELs) have attracted increasing attention $(14,15)$ because they are expected to surpass conventional blue nitride LDs in many applications. In particular, the use of two-dimensional arrays of blue VCSELs would drastically reduce the read-out time in high-density optical storage (compact disc and digital video disc) and increase the scan speed in high-resolution laser printing technology (14). The short vertical cavity configuration is also especially suitable for reducing the threshold current in blue lasers, because wide band gap materials have large optical gain due to the giant joint density of states $(1,2)$. In addition, field patterns from VCSELs are naturally completely circular, whereas the aspect

${ }^{1}$ Institute of Industrial Science, University of Tokyo, 7-22-1 Roppongi, Minato-ku, Tokyo 106-8558, Japan. 'Lehrstuhl für Technische Physik, Universität Würzburg, Am Hubland, 97074 Würzburg, Germany. ${ }^{3}$ Consiglio Nazionale delle Ricerche, Istituto Studio Materiali per l'Elettronica, Universita' di Lecce, via Arnesano 73100 Lecce, Italy. ${ }^{4}$ Istituto Nazionale Fiscica Materia, Universita' di Lecce, Dipartimento Ingegneria Innovazione, via Arnesano 73100 Lecce, Italy.

*To whom correspondence should be addressed. Email: someya@iis.u-tokyo.ac.jp ratio between vertical and horizontal modes is still 4 in conventional edge-emitting nitride lasers $(10)$.

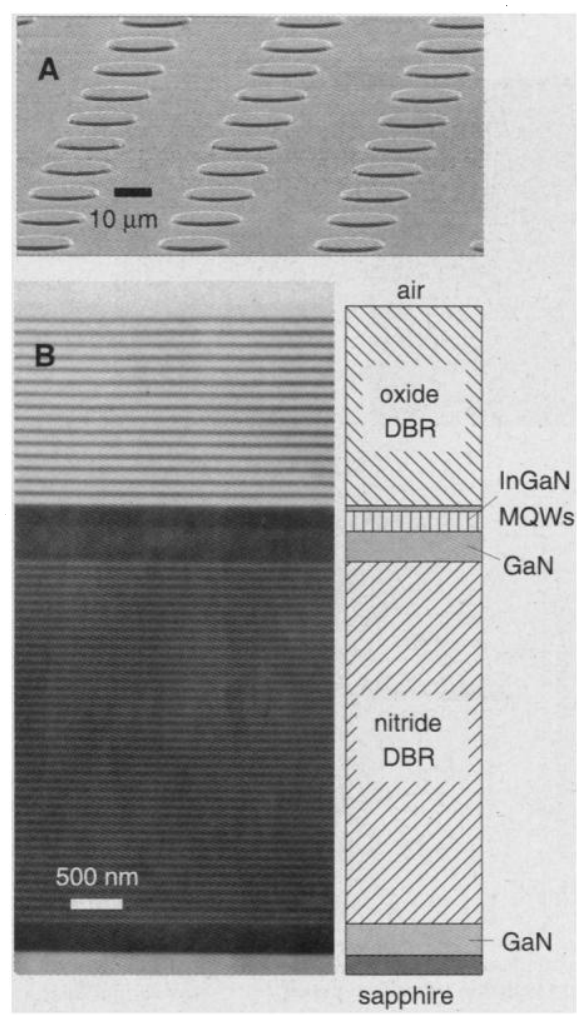

The main obstacle to the room-temperature operation of blue VCSELs is the crystal growth of highly reflective nitride mirrors consisting of $\mathrm{GaN}$ and $\mathrm{AlN}$ or $\mathrm{GaN}$ and $\mathrm{Al}_{x} \mathrm{Ga}_{1-x} \mathrm{~N}$ with high aluminum content $x$ on which the highquality InGaN active regions are subsequently grown $(16-19)$. The problems that arise in epitaxial growth of high-quality films are the large difference in thermal expansion coefficients between $\mathrm{GaN}\left(5.6 \times 10^{-6} / \mathrm{K}\right)$ and $\mathrm{AlN}(4.2 \times$ $10^{-6} / \mathrm{K}$ ) and the large difference in their lattice constants $(2.7 \%)(20)$. However, progress in crystal growth technology is now opening the door to the synthesis of highly lattice-mismatched nitride semiconductor systems.

We report here the fabrication of blue nitride VCSELs and the observation of lasing action at room temperature. Using a microcavity with a high $Q$ factor of 500 , we observed lasing action at a wavelength of 399 nm under optical excitation.

Disk-shaped VCSEL structures $18 \mu \mathrm{m}$ in diameter are formed from a planar multilayer by reactive ion etching and arrayed in a two-

Fig. 1. (A) Scanning electron microscope image of a two-dimensional array of GaN-based VCSELs. (B) Cross-sectional image of the VCSEL structure observed by transmission electron microscopy. The structure of the GaN-based multilayer, grown on a (0001)-oriented sapphire substrate, is as follows: a $30-\mathrm{nm} \mathrm{GaN}$ nucleation layer, 400-nm GaN, a nitride distributed Bragg reflector (DBR) consisting of 43 pairs of 38-nm GaN (dark layers) and 40-nm $\mathrm{Al}_{0.34} \mathrm{Ga}_{0.66} \mathrm{~N}$ (bright layers), 195-nm GaN, multiple quantum wells (MQWs) consisting of 26 periods of $5-\mathrm{nm} \ln _{0.01} \mathrm{Ga}_{0.99} \mathrm{~N}$ barrier and 3-nm $\ln _{0.1} \mathrm{Ga}_{0.9} \mathrm{~N}$ quantum well, and $18-\mathrm{nm}$ $\mathrm{GaN}$. The aluminum content $x=0.34$ of the $\mathrm{Al}_{x} \mathrm{Ga}_{1-x} \mathrm{~N}$ was determined by $\mathrm{x}$-ray diffraction measurements. The oxide DBR consisting of 15 pairs of $48-\mathrm{nm} \mathrm{ZrO}_{2}$ (dark layers) and 68-nm $\mathrm{SiO}_{2}$ (bright layers) was evaporated on the top of the GaN-based multilayer to form a vertical cavity. 
dimensional matrix with $22-\mu \mathrm{m}$ spacing (Fig. 1A). Laser emission from the vertical cavity comes out normal to the plane of the wafer.

As may be seen in the cross-sectional view of our VCSEL structure (Fig. 1B), a $2.5-\lambda$ cavity containing $\operatorname{In}_{0.1} \mathrm{Ga}_{0.9} \mathrm{~N}$ multiple quantum wells as the active region is sandwiched between two highly reflective mirrors: the nitride-based distributed Bragg reflector (nitride DBR) (16-19) and an oxidebased one (oxide DBR). The peak reflectivities were $98 \%$ and $99.5 \%$ for the nitride and oxide DBRs, respectively. The nitride layers were grown by metal organic chemical vapor deposition, and the oxide layers were formed by electron-beam evaporation. The technical details of the growth procedure have been reported elsewhere $(15,16)$.

Spontaneous emission of a single VCSEL was measured at room temperature (Fig. 2A). The beam from a He-Cd laser $(\lambda=325 \mathrm{~nm})$ was incident normally on the sample surface and focussed to a $20-\mu \mathrm{m}$-diameter spot with an objective lens, and emitted light was collected by another objective lens from the backside of the sample. The laser spots and the emission images were monitored with a color chargecoupled device camera. A typical microscopic emission image from the single VCSEL below threshold is shown in Fig. 2B

The spontaneous emission spectrum of the VCSEL below threshold (Fig. 2C) was modified by microcavity effects. The main peak at $\lambda=399 \mathrm{~nm}$ is a cavity resonance mode, and the two other peaks at $\lambda=389 \mathrm{~nm}$ and $408 \mathrm{~nm}$ are due to modulation produced by the transmission spectrum of the nitride DBR. The small bandwidth of $14 \mathrm{~nm}$ is due to the small difference of refractive index between $\mathrm{GaN}$ and $\mathrm{Al}_{034} \mathrm{Ga}_{066} \mathrm{~N}$, that is, about 0.12 . However, this bandwidth is comparable with or even larger than the typical spectral linewidth of luminescence from InGaN quantum well structures.

The spectral linewidth of the cavity mode may be as narrow as $0.8 \mathrm{~nm}$ (Fig. 2C), giving a $Q$ factor of 500, which corresponds to a cavity with $98 \%$ reflecting mirrors, neglecting internal losses. This value is in good agreement with the independently measured reflectivities of the nitride and oxide DBRs, which were $98 \%$ and $99.5 \%$, respectively. We estimate that the optical gain of the InGaN quantum well at the threshold is $g_{\text {th }}>1 /\left(2 L_{\mathrm{w}} N\right) \ln \left(R_{1} R_{2}\right)=890$ $\mathrm{cm}^{-1}$, where $L_{\mathrm{w}}$ is the thickness of the quantum wells, $N$ is the number of wells, and $R_{1}$ and $R_{2}$ are the reflectivities of the DBRs. The estimated gain is comparable with the optical gain of InGaAs-GaAs quantum wells (21).

The dependence of the laser output power on input excitation power was measured at room temperature. As an excitation source we used a dye laser (BPBD365) with a peak wavelength of $367 \mathrm{~nm}$, pumped by a pulsed nitrogen laser with a pulse repetition rate of $3 \mathrm{~Hz}$ and a typical pulse width of 8 to 10 ns. Emission intensities accumulated for 180 pulses as a function of excita-
Fig. 2. (A) Experimental set-up to measure microscopic emission images. (B) Microscopic emission image from a single VCSEL below threshold measured at room temperature, with a He-Cd laser $(\lambda=325 \mathrm{~nm})$ as the excitation source. (C) Spectrally resolved spontaneous emission of (B) The arrow indicates the stop band of the nitride DBR.

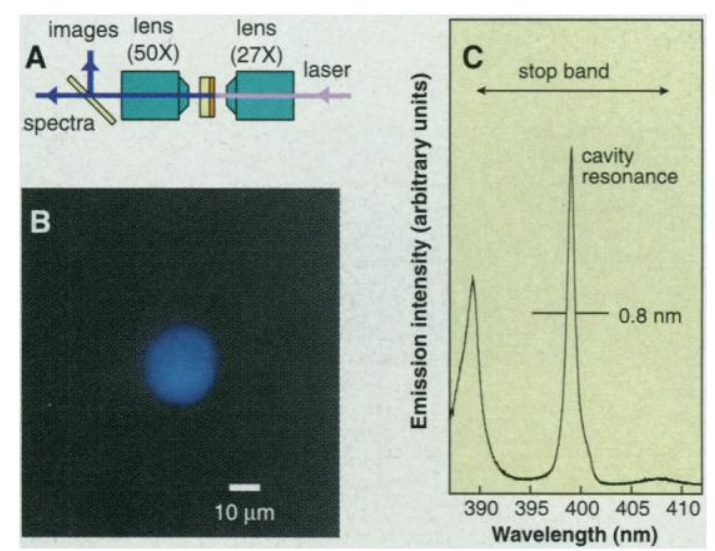

Fig. 3. Emission from a single VCSEL measured at room temperature with an excitation source of a dye laser $(\lambda=367 \mathrm{~nm})$, pumped by a nitrogen laser. (A) Emission intensities accumulated for 180 pulses are plotted as a function of excitation energy per pulse. The incidence and detection directions are opposite to those used for the spectrum presented in Fig. $2 \mathrm{C}$ to avoid degradation of the oxide DBR by illumination with the high-power excitation source. (B) Emission spectra from the VCSEL at various excitation powers $\left(E_{t h}=43 \mathrm{~nJ}\right.$ threshold energy). tion energy per pulse (Fig. 3A) show that a clear threshold is observed at a pump energy of $E_{\text {th }}=$ $43 \mathrm{~nJ}$, which corresponds to $10 \mathrm{~mJ} / \mathrm{cm}^{2}$. Assuming a reflectivity of the nitride DBR at $367 \mathrm{~nm}$ of $50 \%$, absorption in quantum well layers of $50 \%$, and quantum efficiency of $10 \%$, we estimate the sheet carrier density at threshold to be $2 \times 10^{12}$ to $4 \times 10^{12} \mathrm{~cm}^{-2}$.

In previous reports on optically pumped nitride lasers, nonlinear input-output characteristics similar to those shown in Fig. 3A were often confused with lasing action. In some cases, the emission intensity was weak under lowpower excitation because of nonradiative recombination. In other cases, amplified spontaneous emission was observed instead of laser emission. Although spectral narrowing is also observed in amplified spontaneous emission, the typical linewidth of such emission spectra is a few nanometers, which is still much broader than that of laser emission. High-resolution measurements of the emission spectra (Fig. 3B) from the VCSEL for increasing excitation powers show a transition from the 0.8nm-width spontaneous emission peaks spectrally filtered by the microcavity below threshold to very sharp emission peaks above threshold. Note that the linewidth of these sharp peaks is less than $0.1 \mathrm{~nm}$, which is the resolution limit of the detection system. Such a spectral narrowing is direct evidence of lasing action.

\section{References and Notes}

1. S. Nakamura, Science 281, 956 (1998)

2. _ and G. Fasol, The Blue Laser Diode (Springer, Berlin, 1997)

3. S. Nakamura et al., Jpn. J. Appl. Phys. 35, L74 (1996). 4. K. Itaya et al., ibid., p. L1315.

5. S. Nakamura et al., Appl. Phys. Lett. 69, 4056 (1996). 6. Y. Kimura et al., Jpn. J. Appl. Phys. 37, L1231 (1998).

7. G. E. Bluman et al., Electron. Lett. 33, 1556 (1997).

8. M. P. Mack et al., MRS Internet J. Nitride Res. 2, Article 41 (1997). Available at http://nsr.mij.mrs.org/2/41

9. S. Nakamura et al., Jpn. J. Appl. Phys. 37, L1020 (1998).

10. S. Nakamura et al., ibid., p. 1309.

11. I. Akasaki et al., Electron. Lett. 32, 1105 (1996)

12. H. Katoh et al., Jpn. J. Appl. Phys. 37, L444 (1998).

13. A. Kuramata et al., ibid. 36, L1130 (1997).

14. K. Iga, Proceedings of the First International Symposium on Blue Lasers and Light Emitting Diodes Th-11, Chiba, Japan, 5 to 7 March 1996 (Ohmsha, Tokyo, 1996).

15. T. Someya et al., Jpn. J. Appl. Phys. 37, L1424 (1998).

16. T. Someya and Y. Arakawa, Appl. Phys. Lett. 73,3653 (1998).

17. J. Fritz and T. J. Drummond, Electron. Lett. 31, 68 (1995).

18. T. Shirasawa et al., J. Cryst. Growth 189/190, 124 (1998).

19. J. M. Redwing et al., Appl. Phys. Lett. 69, 1 (1996).

20. S. Strite and H. Morkoc, J. Vac. Sci. Technol. B 10, 1237 (1992).

21. L. A. Coldren and S. W. Corzine Diode Lasers and Photonic Integrated Circuits (Wiley, New York, 1995).

22. This work is partly supported by the Japan Society for the Promotion Science, a Grant-in-Aid for Scientific Research from the Ministry of Education, Science, Sports, and Culture, and University-Industry Joint Project on Quantum Electronics. AF. and R.C. thank the Bayerische Forschungsstiftung (G Forschungsstiftung (Germany) and INFM-Progetto Sud (Italy), respectively, for partial financial support.

19 May 1999; accepted 23 July 1999 